\title{
Cheek by Jowl: Reframing Complicity in Web-Streams of Measure for Measure
}

\section{Peter Kirwan}

\section{Renegotiating Liveness}

As I write this paragraph on 13 March 2017, the landing page of Cheek by Jowl's website features a bright orange countdown timer, temporarily overwhelming the rest of this theatre company's web presence (Cheek by Jowl 2017). The countdown stands at 37 days, 2 hours, 33 minutes and 55 seconds; at 7.30pm on 19 April, the Event - a live online stream of a performance of the company's current production of The Winter's Tale, broadcast from the Barbican - will begin.

The countdown timer is the quintessential marker of online liveness in an age of e-commerce, and one that visualizes real time in order to create anticipation and even tension. The slow countdown of the online timer creates a pressured, delimited temporal environment that positions the viewer in relation to their future rather than their past, establishing an end-point that retroactively casts the present as a period of hiatus, or limbo. When the countdown reaches zero, the viewer shifts from being in a queue to purchasing a ticket, or from waiting to see a production to being a member of an online audience. Potential becomes actuality. The timer invites its audience to envision themselves as part of a future community of participation, and to begin generating that community.

Many cinematic livestreams, including NT Live and Live from Stratford-upon-Avon, use onscreen countdown timers as the audience arrive to take their seats, and again during the interval. These timers are, however, designed for the logistical convenience of a pre-formed audience, helping customers plan their toilet breaks and bar trips, not to help form a community; at my local cinema, the tickets for live broadcasts of theatre productions have usually sold out days in advance, and this pre-configured audience only coheres as a shared presence in the physical environment of the cinema. A theatre production livestreamed over the internet, however, does not have the luxury of advance monetary buy-in. It can hope for an audience many times that of a cinema with finite capacity, but it cannot assume that that audience will show up, nor that they will stay. The 'social opprobrium' that accompanies walking out of a theatre, in Pascale Aebischer's words, is entirely absent when the person is sitting alone at home in front of their computer (Aebischer, forthcoming).

Cheek by Jowl's countdown timer substitutes for the advance purchase of a ticket, generating an online community with a social investment in the live event. The extended build-up allows the company to utilize its social networks and stakeholders to inculcate a collective sense of participation long in advance of the live-stream itself, with the ticking clock rendering the moment of the event newly urgent each time it is viewed. The company sends out repeated reminders on its social media channels, and depends on its supporters to broadcast them further. The invitation is not to buy, but to come together as part of a virtual viewing community. In this way, the company's approach to community generation validates Philip Auslander's observation that the experience of communality in a mediatized environment may be more 'genuinely communal' than that experienced by the physically live audience (2008: 65 ); the engaged, sociable dialogue on social media contrasting with the communally silent conventions of mainstream British theatre. In generating the collective sense of buy-in, Cheek by Jowl builds the groundwork by which a disparate online audience can take shared ownership of, and shared responsibility for, a live virtual event. 
Cheek by Jowl's mission statement has always privileged the sense of immediacy and collaborative frisson that inheres in the quotation from A Midsummer Night's Dream that forms the company's name (Reade 1991: 11). Founded in 1981 by Declan Donnellan and Nick Ormerod, the company has grown to be one of the most critically acclaimed of European touring companies, bringing celebrated fresh takes on perennial favourites and neglected gems to six continents, and expanding to produce work in Russian and French in addition to English. Despite the company's prestige, however, it has consistently preferred to tour to mid-size venues (including the Silk Street Theatre at its UK base, the Barbican, rather than the much larger main house), insisting on the importance of 'intimacy and communication' between audience and company and prioritising the quality of the work (Matthews 1995). Further, as the company's international reach has grown, the number of performances it is able to offer in any one country has diminished, limited by the costs of touring. The result can be that watching a Cheek by Jowl production live is a privilege, aligning the company and audience as fellow travellers who have often come some way to share the experience of being guests at a host venue.

Although Donnellan and Ormerod directed their first feature film, Bel Ami, in 2012, it was watching a cinematic broadcast of a recording of their 1991-1995 As You Like It in 2014 that convinced them that the appetite and technology for properly remediating their theatrical productions was there. Choosing a free live-stream rather than a commercial model is a logical decision for a company who wishes to broaden and diversify its audiences, particularly in younger age brackets, while acknowledging the prohibitive costs of both touring and attending a production. The free live-stream also mitigates the company's longstanding policy of limiting the number of school tickets available for each production by offering an alternative mode of viewing, and the liveness of social media and online events can be used to generate excitement among a much broader and internet-savvy crowd. ${ }^{1}$ By beginning with two productions in languages other than English, the Russian-language Measure for Measure and the French-language Ubu Roi, the company opened up its work for potential audience members to try for free, in the hope that those who discovered the company's work through this medium would be more encouraged to make the journey for the in-person experience on future tours. However, the choice of an internet live stream - however much the countdown timer emphasized the importance of the live event - decentres the shared experience of the physical space hitherto at the heart of the Cheek by Jowl ethos. Not only is the viewer of the stream invited to watch the production alone, but the choice of the small(est) screen - the computer, tablet or phone, as opposed to the cinema - risks jeopardising the scale and effect of a company style that is rooted in a distinctively theatrical use of the whole ensemble. Cheek by Jowl's work foregrounds the body of the actor and the spatial relationships between actors; Ormerod's design ('expressive minimalism ... a visual poetry of suggestion', Rutter 2005: 347) emerges in response to, rather than preceding, the rehearsal process, and Donnellan's direction treats space as abstract and fluid, the empty space between actors as significant as that which is occupied.

This chapter focuses on the first of the company's live-streamed productions, Measure for Measure, to consider how the livestream affects the company's aesthetic. This production offers a unique opportunity for comparative analysis as two different versions of the livestream survive: the production as originally broadcast, directed and live-mixed by Thomas Bowles, and a second version re-edited by Donnellan and Ormerod for later use, including as part of an interactive education pack. ${ }^{2}$ This remixed version - importantly, of the same performance - prioritizes different elements of the production, allowing for two similar but importantly different screen versions. ${ }^{3}$ For a production with such political bite, particularly in its acknowledgement of the potential complicity of its audience in witnessing scenes of stage aggression, the two versions also invite consideration of Martin Barker's response to Catherine Belsey's concerns about the 'forced perspective' of screen viewing, which 
Barker argues livecasting can resist (Barker 2013: 50). By reading selected scenes closely, this chapter will unpack the ways in which the two different versions capture empty space, prioritise different characters' subjectivity and ultimately reflect on the live audience's complicity, indicating some of the ways in which the editors of the live camera footage utilize the active potential of liveness. ${ }^{4}$

\section{Framing empty space}

Cheek by Jowl's Measure for Measure was first staged at Moscow's Pushkin theatre in 2013, and was the first Russian-language production directly produced by Cheek by Jowl, following previous successful collaborations with the Maly Theatre and the Chekhov International Theatre Festival. It had toured to Spain, France and Estonia before arriving at the Barbican, where the live broadcast was filmed in partnership with the Roundhouse on 22 April 2015, for the relatively low cost of c. $f 15,000$, and broadcast via the company's own website and The Telegraph, a dissemination partnership in which no money changed hands. The company's decision to begin its live streaming experiments with a Russian-language production already electronically remediated in its live setting via surtitle screens offered a bold statement about Cheek by Jowl's identity as a multilingual company. Measure for Measure was celebrated long before its arrival in London, with reviewers across Europe repeatedly turning to the resonances with Putin's Russia, 'anatomised' in the show (Cheek by Jowl 2015). Performed in contemporary dress, the production explored the severity of a state clamping down on deviant sexuality, the moral complexity of a ruler who watches everything and manipulates his own homecoming, and the compromised choices of Isabella, captured in the poignant final scene as brother Claudio gave her the cold shoulder, cradling his wife and child while Isabella looked on tearfully.

This emotional and political impact was dependent on a visual structure that enabled watching and reacting. A central pillar of Cheek by Jowl's style is the presence and participation of the whole ensemble; indeed, in productions as recent as Macbeth (2009-11) and 'Tis Pity She's a Whore (2011-2014), the majority of the company remained visible onstage for the duration of the performance. Members of the Cheek by Jowl ensemble move fluidly between named characters, unmarked bodies, and a liminal state in which actors present their characters while keeping them spatially detached from the scene in question, often responding visibly to scenes that they are not active in. In these productions, characters are constantly watching, a state particularly apt for Measure for Measure, a play constructed around the Duke's surreptitious surveillance of his people. Especially at the production's start, a tightly knit body of characters silently watched the action, leading to the stage's construction around a tripartite structure of gazing: the characters within a given scene at one another; the onstage chorus at the primary scene; the audience watching both scene and chorus. For much of the production, the disguised Duke (Alexander Arsentyev) joined the Chorus in order to watch events (often in horror) regardless of his literal presence in a scene. When I first saw the production in Moscow, the Duke's constant presence as both participant and observer rendered him quite clearly (to me, at least) the central character of the production. ${ }^{5}$

In the Silk Street Theatre at the Barbican, the company utilized the full width and depth of the stage, providing a cavernous environment for the different groups onstage to watch one another, often over large distances; as Christopher Innes and Maria Shevtsova put it, 'all the better to see and be seen, the eyes leading to what is at stake between them' (2013: 213). The wordless opening sequence, which established the Duke's spatial relationship to the chorus within Ormerod's 'expressive minimalism', offered a particular challenge to the cameras in how it used a great deal of empty space, and the extreme differences in the two versions of the stream are instructive. The production was filmed using four cameras, as follows (the designations of letters to cameras are my own):
Commented [anon21]: Not entirely clear how the footnote relates to the argument here.

Commented [PK2R1]: Have incorporated into endnote 2. 


\begin{tabular}{|l|l|}
\hline Camera A & $\begin{array}{l}\text { Situated at the very top and back of the raked } \\
\text { audience seating, capturing a wide angle } \\
\text { overview in which audience members were } \\
\text { visible. }\end{array}$ \\
\hline Camera B & $\begin{array}{l}\text { Dead centre of downstage, within the audience } \\
\text { and almost at stage level. }\end{array}$ \\
\hline Camera C & $\begin{array}{l}\text { At stage level in front of the live audience, at } \\
\text { extreme stage left }\end{array}$ \\
\hline Camera D & $\begin{array}{l}\text { At stage level in front of the live audience, at } \\
\text { extreme stage right }\end{array}$ \\
\hline
\end{tabular}

The four cameras evoked a range of the potential perspectives from which audience members in the theatre could view the production, as well as showing part of the theatre audience, doing some virtual community-building for the viewer at home. None of the cameras appear to have had the ability to move, though C and D could pan and zoom. The proximity of stage and audience in this studio theatre, where front-row audience members have their feet on the stage, meant that cameras $C$ and $D$ were often shooting at an almost perpendicular angle to the actor-audience axis when following actors downstage.

Measure for Measure opened to a set comprised of five red cubes, three in a horizontal line bisecting the stage at roughly halfway along the upstage-downstage axis, and two at extreme stage left and right, a little downstage of the others. The five cubes formed a downstage playing space, about half the area of the whole stage, brightly lit by hanging vertical lamps whose beams could be seen against the surrounding blackness. During this opening sequence, a group of individuals, closely packed, appeared between two of the cubes. The group moved around the stage in a sequence of choreographed positions until Arsentyev's Duke was produced from within the ensemble and separated from them. He then moved independently while the group responded to him; he seemed at times to flee the group and at others to be coaxed into sitting on a chair. The sequence used the whole width and much of the depth of the stage.

On the live stream, the dominant viewpoint in this extract is that of Camera A, used for 2 minutes and 14 seconds of the whole sequence (about half of the total 5 minutes and 7 seconds from lights rising to the first line of dialogue). This wide angle captures the shape of the entire stage space, doing the work identified by Susanne Greenhalgh for 'cinema audiences [who] need the theatrical space and what it symbolizes to be clearly delineated ... [with the] juxtaposition of wide or high angle establishing shots' (2014: 259) and here emphasising the distinctive Cheek by Jowl interplay of bodies and space. Actors' faces are not distinguishable for large portions of the sequence, and the overall effect is of a body splitting away from a mass. This individual body is positioned constantly in relation to the mass, making visible the gulf of space between them at certain moments. The 'strange formality' of the wide shot, and the palpable anxiety of the Duke, allow for an emblematic reading of the figure isolated from the pack, produced by but newly separate to the people (Barker 2013: 15).

By contrast, Camera A is entirely absent from the remixed feed, which prioritizes the offcentre angles of Cameras $C$ and $D$ that give medium and close shots of the actors' faces, with regular reference to Camera $B$ to indicate the broad shape of the stage. The whole stage space is never visible: Camera B only shows the three central red cubes, which makes the angles from $C$ and $D$ disorienting at times, as they regularly frame actors at almost exact right angles to $B$ against the otherwise unseen cubes at the far sides of the stage, resulting in what Erin Sullivan refers to as 'a series of visually disconnected zones that can be difficult for remote audiences to imagine back together' (Sullivan, 
forthcoming). The cameras instead work to fill the frame with bodies and colour, as opposed to the black empty space that fills Camera A. The difference between the two versions is most apparent as the Duke and Chorus react to one another across the width of the stage. The Duke is at extreme stage left, the Chorus at stage right. They move upstage and then downstage together, mirroring one another's movements. The Chorus then begins crawling across the floor towards him; he paces and keeps his distance. In the live version, Camera A's view emphasizes the gulf between the two groups and the symmetry of their movements. The remixed version, however, cuts between Cameras $\mathrm{C}$ and $D$ in a standard angle/reverse-angle pattern, alternating the views of the Duke and Chorus as they react to one another, privileging their emotional reactions (confusion and fear for the Duke, hopeful expectation for the Chorus). The remixed version of this opening sequence is far more in keeping with screen conventions, with its privileging of facial performance, relative close-up and emotional reaction. The livestream retains something more of the theatrical experience with its constant reminders of the entire mise-en-scène, its insistence on establishing the actors within delimited space, and the emphasis on blocking and symmetry over individual performance. The remix reflects the transition of this product from theatre to live broadcast to edited theatrical film, remediating liveness for the web viewer and education pack user, and in doing so the production conceptually shifts; the 'gain' is the access to expression and internal conflict from multiple potential perspectives; the 'loss' is the stage environ that makes formal sense of the blocking.

In the first few minutes of Cheek by Jowl's first live broadcast, then, a tension arises between the company's hallmark use of empty space and the ability of the screen to capture that space. In a Cheek by Jowl production, abstracted space disrupts realistic conceptions of human interaction; for instance, when placing characters having an intimate conversation at opposite ends of the stage, talking to each other as if in direct proximity, the empty space speaks to the psychic and emotional distance between the two. Space is always active in a Cheek by Jowl production, representing the conflicts and barriers between people. The early spatial separation of the Duke and Chorus is significant given the close bond with which they began; yet in the remixed version, with its preference for 'more insistent mediations of multiple camera shots framed tightly on individuals and small groups and the editing between these' (Wyver 2014: 106), epitomized in the cross-cutting between Duke and Chorus, the active space disappears. They are still clearly separate, but the precision and fluidity of that separation disappears in editing.

\section{Framing the subject}

While the livestream may have captured more of the spatial dynamics of this opening sequence, it makes for poor screen entertainment, especially for an audience unused to fixed-camera archive recordings. The relatively static screen and depersonalized character movements of the livestream are replaced by close attention to expression and reaction in Donnellan and Ormerod's re-edit, providing direct access to the emotional stakes of the scene: a decision that both takes advantage of the opportunities provided by close-ups, and makes the remixed version more useful to the education pack with which it is paired, which includes several activities and interviews focused on character. ${ }^{6}$ Yet the choices of which cameras capture the action have the potential to subtly but significantly change the meaning of the production, as for example in the case of the introduction of Andrei Kuzichev's Angelo.

The opening wordless sequence ends with the Duke standing with Escalus, and the two of them engage in an edited version of the opening dialogue of 1.1, at which point both filmed versions align more closely, prioritising a close shot from Camera $C$ that frames the two characters entirely. The livestream, however, includes a cutaway to Camera A, which reveals the position of the Chorus at stage right (where they have presumably been standing throughout the conversation) watching the 
two; the remixed version remains focused on the diegetic scene between the Duke and Escalus. When the production reaches Escalus's lines

If any in Vienna be of worth

To undergo such ample grace and honour,

It is Lord Angelo. (1.1.22-4)

the Chorus jogs across to Stage Left, sweeping Escalus with them and leaving the Duke alone centrestage, holding out his hand to the newly-absent Escalus. The Duke then removes his glasses to wipe them. In the livestream, Camera B captures the whole sideways movement, including Escalus being caught up as if in a tide by the jogging ensemble. A cut to Camera $D$ shows the reverse angle, with the Chorus now at stage left looking back at the Duke; then, a dramatic cut to Camera A reveals that another lone figure has been left at stage right: Angelo, dressed identically to the Duke, who has been revealed from within the Chorus exactly as the Duke was earlier. The Duke starts when he notices him.

In the remixed stream, this whole sequence shifts to privilege the Duke's perspective. On 'It is Lord Angelo', the sound of the jogging is heard, but the shot stays with Camera C. The Chorus suddenly enter the frame from nowhere and move through it; while the sweeping away of Escalus remains visible, it is the Duke's reaction that is central. The shot then shifts to Camera D (as with the live feed), but stays with Camera $D$ until he turns and jumps. Only then does a cut to Camera $C$ reveal Angelo in a medium shot, framed against the red background of one of the cubes. For viewers of the remixed version, Angelo's location on the stage is unclear.

The differences here offer a fundamental recategorization of theatrical space, to extraordinary effect. The livestream offers a reading of the scene as an emblematic transfer of power in the stage space. In the play, Escalus and the Duke talk and Angelo enters to them. In Cheek by Jowl's version, the entire spatial organization of the stage switches to indicate a change of focus, or 'target', to borrow Donnellan's preferred term for an actor/character's objective (Donnellan 2005: 16-29). The Chorus sweeps across the stage space like a pendulum, replacing the actor playing Escalus with the isolated figure of Angelo, to whom the Duke now turns. Further, Angelo is present and distinguishable long before the Duke reacts to his presence, creating a visual dramatic irony that contributes to the ongoing nervousness of the Duke. In the remixed version, Cameras $C$ and $D$ focalize the scene through the Duke's perspective. Camera $\mathrm{C}$ is predominant while the Duke and Escalus talk, showing the Duke's full face but the back and side of Escalus's head. The movement of the Chorus across the stage is effectively non-diegetic, the camera ignoring it to stay on the Duke. Then, only once the Duke is aware of Angelo's presence does the camera show him.

As with the earlier elision of active empty space, Donnellan and Ormerod's filmic choice in the remixed version to conceal Angelo until the Duke sees him operates against another recurring feature of their theatrical practice. Cheek by Jowl's dramaturgy highlights characters when they are spoken about as well as when they are literally present in a scene, what Innes and Shevtsova refer to as 'tableau-freeze ... signifying that they belong to the whole story of the play', although in recent years the company has shown a preference for a mobile rather than frozen visible ensemble (2013: 215). The remixed version nixes this, substituting the (differently effective) jump cut between two very similarly dressed men, now aligned within screen space (their position within the frame) rather than theatrical space (their symmetrical revelations and blocking). The remixed version re-reads the scene from the Duke's perspective, isolating him within a flurry of movement and disconnecting him from his spatial surroundings. The loss is the symbolic resonance of Angelo's own spatial isolation; while both edits frame him as being alone, the distinction is much less clear and the distance of his physical 
separation is, again, elided in the remix. The gain is the emotional alignment of audience with protagonist, with the viewer invited to share the Duke's surprise at Angelo's unexpected presence.

Something subtler, and perhaps unintentional, is at work in the move from theatrical production to livestream to remixed digital video. Across the three versions, the ensemble and the empty space become progressively less visible, while individual facial expression and subjective experience come to the fore. In the livestream (and, I would argue, the production in the theatre), the dominant visual motif is of subjects being watched; in the remixed version, it is the subjects themselves looking. This is also a movement from the structured politics that position an individual in relation to the state, to the experience of an individual discovering their own subjectivity, as is perhaps most apparent in the first encounter between Angelo and Isabella in 2.2. Pascale Aebischer has written about the ways in which the live broadcast repeatedly 'refused to focus on Isabella at key moments or showed her from the point-of-view of a character in whose eyes she embodied repressed sexual desire rather than chastity', and the partial rehabilitation of this by Donnellan and Ormerod in the remixed version (Aebischer, forthcoming). This is, inevitably, not neat enough for an absolute distinction between versions - in 2.2, for instance, the livestream focuses on Isabella almost without interruption, while the edited version's shot/reverse-shot mode repeatedly turns from her to Angelo - but most of the shifts between livestream and remix contribute to Isabella's experience, including framing her as she waits to be admitted to Angelo, where the livestream lingers on him in close-up. The most important effect of this comes as she sits down. In the remixed version, Camera $C$ frames her as she sits and responds to the unseen Angelo; in the live version, Camera B shows her sitting down in front of him. The live feed gives an overview of the scene of conflict, structured by the theatrical space between the actors; the remixed version prefers her experience of the scene, capturing her subjectivity.

\section{Framing complicity}

These close readings can only be indicative, but cumulatively they offer a version of the performance that, I suggest, lends itself to a readjustment of the production's politics. In these scenes, Bowles's original live edit privileges the Duke, the ensemble and the space of the state, while Donnellan and Ormerod's remixed version privileges Isabella, individual reaction and the personal space. Both versions are partial, each version focusing on aspects deprioritized by the other while offering a coherence relative to its own priorities. In allowing such different readings of the production's interests, I would like to conclude by arguing that the existence of these two edits draws attention to the relative complicity of the audience in the events of the play, particularly within a 'live' - if virtual - environment.

While the remixed version gives much more space and time to Isabella, the Chorus is rendered largely invisible. In the theatre, the constant presence of the Chorus was a defining aspect of the production, stressing the surveillance state and always showing the reactions of those affected by the main action. 'The space is not neutral', to borrow one of Donnellan's adages, and a character 'cannot do whatever she likes in the space' (Donnellan 2005: 125); in this production, the Chorus of onlookers loads the space with meaning. The dynamic of on-stage watching particularly served the character of the Duke, who was usually at the front of the Chorus when not directly involved in a scene. The shot/reverse-shot structure of the remixed version understandably focuses on the participants in the scene, and thus inadvertently reads the work of the Chorus as reactive, passive, and thus excludable, so deprioritising the role of the Duke.

At the end of 2.2, Angelo delivers his soliloquy revealing his reaction to Isabella. The remixed version shows this by alternating between Cameras B and D, framing Angelo as by himself - as indeed 
the scene calls for him to be. Yet the live version includes an establishing shot from Camera A that reveals the Chorus are standing at upstage right, watching - and implicitly judging - Angelo as he speaks. When Angelo crosses to stage right during his soliloquy, the remixed version continues to frame him with Camera D, which keeps him in his isolated psychic space, rather than switching to Camera $\mathrm{C}$ which would have framed him against the Chorus. By including or excluding the Chorus, the editors of the screen versions invite very different readings of the speech. However, the remixed version's decision to exclude the Chorus is thwarted by a moment in which Angelo starts fondling and smelling the chair on which Isabella had been sitting, at which point the Chorus crosses to him. The live version, which had already shown the Chorus at the side of the stage, reads this as a moment in which the Chorus are prompted into motion by the extremity of his action. In the remixed version, which remains in close-up on Angelo, he is suddenly and inexplicably joined in the frame by a selection of unannounced feet.

This moment is revealing of the difficulties inherent in translating Cheek by Jowl's use of theatrical space and non-diegetic activity to the screen. The shots of the remixed version read the scene literally, preserving the integrity of the scene's imagined location and Angelo's isolation, but treating the scene in this way does not account for the intervention of the non-diegetic Chorus, unseen by Angelo but intruding in his theatrical space. Cheek by Jowl's visual aesthetic is fluid in the theatre: not only do characters appear and move among scenes that they are not supposed to be participating in, but their scene transitions deliberately overlap dialogue and blocking, so that scenes blur into and comment on one another rather than being demarcated. While the remixed version better captures the emotional arcs of individual characters and scenes, this comes at the expense of a frame that shows a confused image when confronted with the non-diegetic, fluidly theatrical aspects of the production.

Even more importantly, the two versions frame complicity differently, as is most apparent in 2.4. As with 2.2, the livestream makes greater use of Cameras A and B to keep both actors in shot at the same time, framed as sitting together at the same table, whereas the remixed version uses a shot/reverse shot format to juxtapose Angelo and Isabella as they speak (and, as Aebischer [forthcoming] points out, to better privilege Isabella's experience of the scene). More significant, however, is the divergence in the treatment of direct address. When Angelo asks Isabella 'Who will believe you?' (2.4.153), ${ }^{7}$ he gestures downstage. The remixed version stays focused close on the two actors, but in the livestream the shot cuts quickly to Camera A, showing and thus implicating the theatre audience as the 'Who', and making clear their silence. Subsequent to this, Angelo moves to centre stage, sits in a chair and unbuckles his belt. The remixed version continues capturing this using Camera B, showing the space between the two actors and framing this as a private moment; the live stream uses Camera A, not only continuing to implicate the audience as silent bystanders, but more pointedly bringing the Chorus back into the shot. The attempted rape that follows is a private scene of trauma in the remixed version, but the livestream invites the viewer to note the silent observation of this by two separate groups of onlookers. Following the assault, as Isabella begins her soliloquy 'To whom should I complain?' (2.4.171), the livestream again cuts out to Camera A, which not only puts pressure on the theatrical and implicitly the web audience to identify themselves as the 'whom', but also shows Angelo sat back at his desk, nonchalantly going through his papers as if nothing has happened. The remixed version, shown almost entirely from Camera C, isolates Isabella against blackness, removing her from both the subject of her monologue and from her audience, suggesting that she is indeed alone in her complaint. The remixed version, I suggest, better captures the pathos of Isabella's cry; the livestream better holds to account the abusers and those rendered complicit in the abuse by their silence.

Commented [anon23]: In the footnote, add the line number and include the Arden reference in your works cited list.

Commented [PK4R3]: Done, but I rather assumed we'd be using the Arden Complete Works as a standard, which I be using the Arden Complete Works as a standard, which
don't have a copy of. For now I've just used the Arden 2 $M 4 M$, if that's okay? 
It is on this last point that I wish to close by returning to the value of liveness, which in the context of these two versions of the same production allows for the challenge to Belsey's 'forced perspective' that Barker believes the livecast can offer (Barker 2013: 50). The remixed version of this production was designed for use after the event, primarily as part of the company's education package. While a real-time document of a live performance, therefore, it was never watched live; its presence was a construct of the past, its actors locked into a mechanically reproduced moment. Its focus on emotional truth as captured in the faces of actors is better suited to the recorded medium it occupies. The live webcast, by contrast, carries with it - as do all live broadcasts - the spectre of the unknown, the possibility of the unexpected. The unexpected is usually imagined as the potential for failure, the frisson of knowledge that something may go wrong. But the evocation of complicity and the implicit judgement of a silent audience speaks to something that only resonates as part of the live event, in which it remains possible (if highly unlikely) that an intervention could happen and the assault could be stopped, that something could go right, from the perspective of social responsibility if not theatre etiquette. The livestream acknowledges the choice I have made to participate in a virtual, real-time community by foregrounding, at these moments, the presence and silence of the physical audience, an audience that is dispersed in the version released for post-event viewing, but with whom I share complicity in the live moment. The choice to be part of a live event is also the choice to be accountable.

\section{Bibliography}

Aebischer, P. (forthcoming), Shakespeare, Spectatorship and Technologies of Performance, Cambridge: Cambridge University Press.

Auslander, P. (2008), Liveness: Performance in a Mediatized Culture, $2^{\text {nd }}$ ed., London: Routledge.

Barker, M. (2013), Live to Your Local Cinema: The Remarkable Rise of Livecasting, Basingstoke: Palgrave Macmillan.

Cheek by Jowl (1990), poster for Box Office staff promoting Hamlet, V \& A archives THM 24/2/12.

Cheek by Jowl (2015), 'Measure for Measure: Reviews', Cheek by Jowl. Available online: http://cheekbyjowl.com/measure for measure.php\#reviews (accessed 21 April 2017).

Cheek by Jowl (2017), 'Home', Cheek by Jowl. Available online: http://www.cheekbyjowl.com (accessed 21 April 2017).

Davis, E. (2015), 'Measure for Measure screenplay', Cheek by Jowl. Available online: http://archive.cheekbyjowl.com/wp-content/uploads/2015/04/Screenplay.pdf (accessed 21 April 2017).

Donnellan, D. (2005), The Actor and the Target, rev. ed., London: Nick Hern Books.

Greenhalgh, S (2014), ‘Guest Editor's Introduction', Shakespeare Bulletin, 32 (2): 255-61.

Innes, C. and M. Shevtsova (2013), The Cambridge Introduction to Theatre Directing, Cambridge: Cambridge UP.

Matthews, B. (1995), Fax Correspondence, 28 April, V \& A archives THM 24/2/17.

Reade, S. (1991), Cheek by Jowl: Ten Years of Celebration, Bath: Absolute Classics.

Commented [anon25]: Not clear what the corresponding bit is in the text. If it's not cited, cut. If it's cited and the reference is unclear, please clarify.

Commented [PK6R5]: It's in Endnote 1 - I can't see how I could make this any clearer? 
Rutter, C. C. (2005), 'Maverick Shakespeare', in B. Hodgdon and W. B. Worthen (eds), A Companion to Shakespeare and Performance, 335-58, Malden, MA: Blackwell.

Shakespeare, W. (1967), Measure for Measure, ed. J. W. Lever, London, Bloomsbury.

Sullivan, E. (2017), "'The forms of things unknown": Shakespeare and the Rise of the Live Broadcast', Shakespeare Bulletin. 35.4: ADDPAGE NUMBERS WHEN KNOWN.

Wyver, J. (2014), “'All the Trimmings?": The Transfer of Theatre to Television in Adaptations of Shakespeare Stagings', Adaptation 7 (2): 104-20.

\footnotetext{
${ }^{1}$ The company's policy to limit school groups to $25 \%$ of the total audience, and to split large parties into groups of 4 or 5 around the auditorium, was designed to limit distraction and help younger audience members 'gain a fully adult experience of what going to the theatre is all about' (Cheek by Jowl 1990).

${ }^{2}$ This essay is only possible thanks to the generous support and access provided by Cheek by Jowl, especially Dominic Kennedy and Sarah Fortescue, to both versions. The Cheek by Jowl website includes a 'screenplay' prepared by Emma Davis (2015), assistant producer for the broadcast, which the reader may wish to consult for visual reference. The 'screenplay' is a shorthand camera script for the camera operators, but captures a detailed picture of the production's main blocking.

${ }^{3}$ Camera rehearsals take place about five days before the broadcast, without Ormerod and Donnellan in the room. The directors then re-watch the recording of the camera rehearsal and provide notes to the director for the live stream. The decision to re-edit the live broadcast for later use, including the education pack, was not repeated for the next broadcast production, Ubu Roi, filmed in New York on 26 July 2015. I am grateful to the company's Executive Director, Eleanor Lang, for her insight and information about the project and process.

${ }^{4}$ The Cheek by Jowl website includes a 'screenplay' prepared by Emma Davis (2015), assistant producer for the broadcast. The 'screenplay' is a shorthand camera script for the camera operators, but captures a detailed picture of the production's main blocking.

${ }^{5}$ The Duke was played by Valery Pankov at this performance, 24 November 2014 at the Pushkin Theatre. It is worth noting that I do not speak Russian, and performances in Moscow were obviously not surtitled; this interpretation, therefore, may speak as much to my unusual dependence on the visual dynamics of the production rather than the oral/aural qualities.

${ }^{6}$ The Education Pack is not publicly available, but is provided on request by Cheek by Jowl to school and academic institutions.

${ }^{7}$ Here and elsewhere I use the wording of the on-screen subtitles; Arden records the line as 'Who will believe thee, Isabel?' (2.4.153).
} 